\title{
Weekly teriparatide injections successfully treated advanced bisphosphonate-related osteonecrosis of the jaws
}

\author{
D. Yoshiga • Y. Yamashita $\cdot$ I. Nakamichi • T. Tanaka • \\ K. Yamauchi • N. Yamamoto $\cdot$ S. Nogami • T. Kaneuji • \\ S. Mitsugi • T. Sakurai • H. Kiyomiya • K. Tominaga • \\ Y. Morimoto $\cdot$ T. Takahashi \\ Received: 26 November 2012 / Accepted: 15 January 2013 /Published online: 31 January 2013 \\ (C) The Author(s) 2013. This article is published with open access at Springerlink.com
}

\begin{abstract}
This study investigated whether weekly teriparatide (TPTD) injections are as effective as daily teriparatide injections for the treatment of stage 3 bisphosphonaterelated osteonecrosis of the jaws (BRONJ) and compared serum markers of bone turnover between the two treatment regimens. Daily TPTD treatment has recently been reported to be effective for BRONJ, but there are no reports describing the effectiveness of weekly TPTD injections. We report two patients with stage 3 BRONJ. One patient was successfully treated with weekly TPTD injections and the other with daily TPTD injections. Changes in the levels of serum $\mathrm{N}$-telopeptide of type I collagen (s-NTX) and serum Nterminal propeptide of type I collagen (P1NP) were studied. Two patients with stage 3 BRONJ that was refractory to conservative treatment were treated with TPTD. Their medical records were reviewed and the patients were interviewed. There was complete mucosal coverage of the intraoral defects after 3 months of TPTD treatment in both patients. Progressive bone regeneration in an area of mandibular fracture was identified after 4 months of treatment.
\end{abstract}

D. Yoshiga $\cdot$ K. Yamauchi $\cdot$ S. Nogami $\cdot$ T. Kaneuji $\cdot$ S. Mitsugi

T. Sakurai $\cdot$ H. Kiyomiya $\cdot$ T. Takahashi

Division of Oral and Maxillofacial Reconstructive Surgery,

Department of Oral and Maxillofacial Surgery,

Kyushu Dental University, Kitakyushu, Japan

Y. Yamashita

Section of Oral Surgery, Department of Oral and Maxillofacial

Surgery, Fukuoka Dental College, Fukuoka, Japan

I. Nakamichi

Division of General Internal Medicine, Department of Health

Promotion, Kyushu Dental University, Fukuoka, Japan

T. Tanaka

Division of Diagnostic Radiology, Department of Oral

Diagnostic Science, Kyushu Dental University, Kitakyushu, Japan
The s-NTX level increased slightly in both patients. This is the first report of successful treatment of stage 3 BRONJ with weekly TPTD injections. Either daily or weekly TPTD injections may effectively treat stage 3 BRONJ and should be considered before or perhaps even in lieu of undertaking major resection and reconstruction.

Keywords Bisphosphonate - Osteonecrosis · P1NP . s-NTX $\cdot$ Teriparatide

\section{Introduction}

Nitrogen-containing bisphosphonates (N-BP) are prescribed for the treatment of bone diseases such as osteoporosis, multiple myeloma, cancer metastases, and Paget's disease. However, bisphosphonate-related osteonecrosis of the jaws (BRONJ) has been reported as a rare complication. BRONJ occurs at a much higher rate in patients receiving intravenous $\mathrm{N}$-BPs for cancer treatment versus oral N-BPs. The incidence

\footnotetext{
N. Yamamoto

Department of Oral and Maxillofacial Surgery,

Faculty of Medicine, Oita University, Oita, Japan

K. Tominaga

Division of Maxillofacial Diagnostic and Surgical Science,

Department of Oral and Maxillofacial Surgery,

Kyushu Dental University, Kitakyushu, Japan

Y. Morimoto

Division of Diagnostic Radiology, Department of Oral

Diagnostic Science, Kyushu Dental University, Fukuoka, Japan

T. Takahashi

Division of Oral and Maxillofacial Surgery, Department of

Oral Medicine and Surgery, Tohoku University Graduate

School of Dentistry, Sendai, Japan
} 
of BRONJ in patients treated for osteoporosis is low at $0.1 \%$, but the incidence of BRONJ in cancer patients treated with high doses of intravenous N-BP is higher at 3 to $10 \%$ [1].

Currently, conservative treatment is recommended for BRONJ, in accordance with the American Association of Oral and Maxillofacial Surgeons (AAOMS) Position Paper [2]. Recently, however, it has been reported that daily parathyroid hormone treatment is effective for BRONJ. Weekly teriparatide (TPTD; human parathyroid hormone peptide 1-34) injections have been used to treat osteoporosis in Japan [3], but there are no reports describing the effectiveness of weekly TPTD injections for the treatment of BRONJ. Management of BRONJ is challenging and controversial, and there is currently no established drug treatment for this condition. We report two patients with stage 3 BRONJ. One patient was successfully treated with weekly PTD injections, and the other with daily TPTD injections. Changes in the levels of serum Ntelopeptide of type I collagen (s-NTX) and serum N-terminal propeptide of type I collagen (P1NP) were studied.

\section{Case reports}

\section{Case 1}

An 87-year-old Japanese woman with a 4-year history of alendronate therapy ( $35 \mathrm{mg} /$ week orally) was referred for the treatment of multiple fistulas with purulent discharge over the left maxillary ridge. She was diagnosed with stage 3 BRONJ according to the AAOMS guidelines (2009). She initially received conservative treatment, including instruction on oral hygiene, administration of antibiotics, antimicrobial mouth gargles, and local irrigation. N-BP therapy was discontinued at the time of her first visit. Three months later, she underwent sequestrectomy and extraction of the maxillary left first and second molars because of high tooth mobility (Fig. 1a, d, g). We continued conservative therapy and debridement for 1 year. However, her disease was persistent and progressive (Fig. 1b, e, h). She was then treated with TPTD by subcutaneous injection $(56.5 \mu \mathrm{g}$ weekly). After 3 months of TPTD treatment, there was complete coverage of the necrotic tissue and exposed bone with normal mucosa. Computed tomography showed that her maxillary sinusitis attributed to stage 3 BRONJ had resolved (Fig. 1c, f, i). Her s-NTX levels were slightly higher than the pretreatment level at 1 and 3 months after the initiation of TPTD treatment, and her serum P1NP level decreased after the initiation of TPTD treatment (Fig. 1j).

\section{Case 2}

An 81-year-old Japanese woman with a 5-year history of alendronate therapy ( $35 \mathrm{mg}$ /week orally) was admitted for the treatment of a pathological mandibular fracture. After hospitalization, the teeth of the right mandible were naturally detached after cutting the bridge; consecutively, metal crowns were used. She was diagnosed with stage 3 BRONJ and stopped her alendronate therapy after consultation with her physician. She had infection of both the bone and soft tissues (Fig. 2a, b, c). We advised surgical treatment, but this was refused by the patient and her family. We administered conservative treatment for BRONJ and the mandibular fracture, including infection control and use of a chin cap to limit movement of the jaw. After 2 months, her disease was persistent and the fracture was still mobile. We started TPTD treatment by subcutaneous injection $(20 \mu \mathrm{g}$ per day). Three months later, her symptoms had resolved. The osteonecrosis had healed and was covered by normal mucosa. Computed tomography showed partial healing of the mandibular fracture (Fig. 2d, e, f). Her s-NTX and P1NP levels were low at the first visit. Her s-NTX levels were slightly increased compared with the pretreatment level at 2 and 4 months after the initiation of TPTD treatment, and her serum P1NP level was significantly increased at 2 months after the initiation of TPTD treatment (Fig. 2g).

\section{Discussion}

BRONJ is a rare condition than can be debilitating. The most frequent presentation of BRONJ is a small amount of bare bone that is not painful or inflamed, which may heal quickly, slowly, or not at all. Most cases are not as severe as in the patients presented above. Recently, it has been suggested that N-BP treatment may cause BRONJ [4]. BRONJ is much more frequent in patients receiving intravenous $\mathrm{N}$ BP for the treatment and prevention of cancer-related skeletal conditions than in patients receiving oral N-BP for the treatment of non-malignant disease [5]. BRONJ may be associated with the type and total dose of N-BP treatment, and with a history of trauma, dental surgery, and dental infection [6]. We described an 87-year-old female with stage 3 BRONJ that persisted after control of the bone and soft tissue infections, who required tooth extractions 3 months after the withdrawal of N-BP treatment.

The main effects of N-BP are at the lumbar spine and proximal femur, where they stop bone loss, reduce fracture risk, and increase bone mineral density. Local trauma and infection in the jaw increase the demand for bone repair, which may exceed the low turnover rate of the bone, resulting in the accumulation of necrotic bone that is recognized as osteonecrosis of the jaw.

There are some previous reports of TPTD treatment in patients with osteonecrosis of the jaws associated with N-BP therapy [7-9]. Additionally, several patients treated with daily TPTD injections have now been 

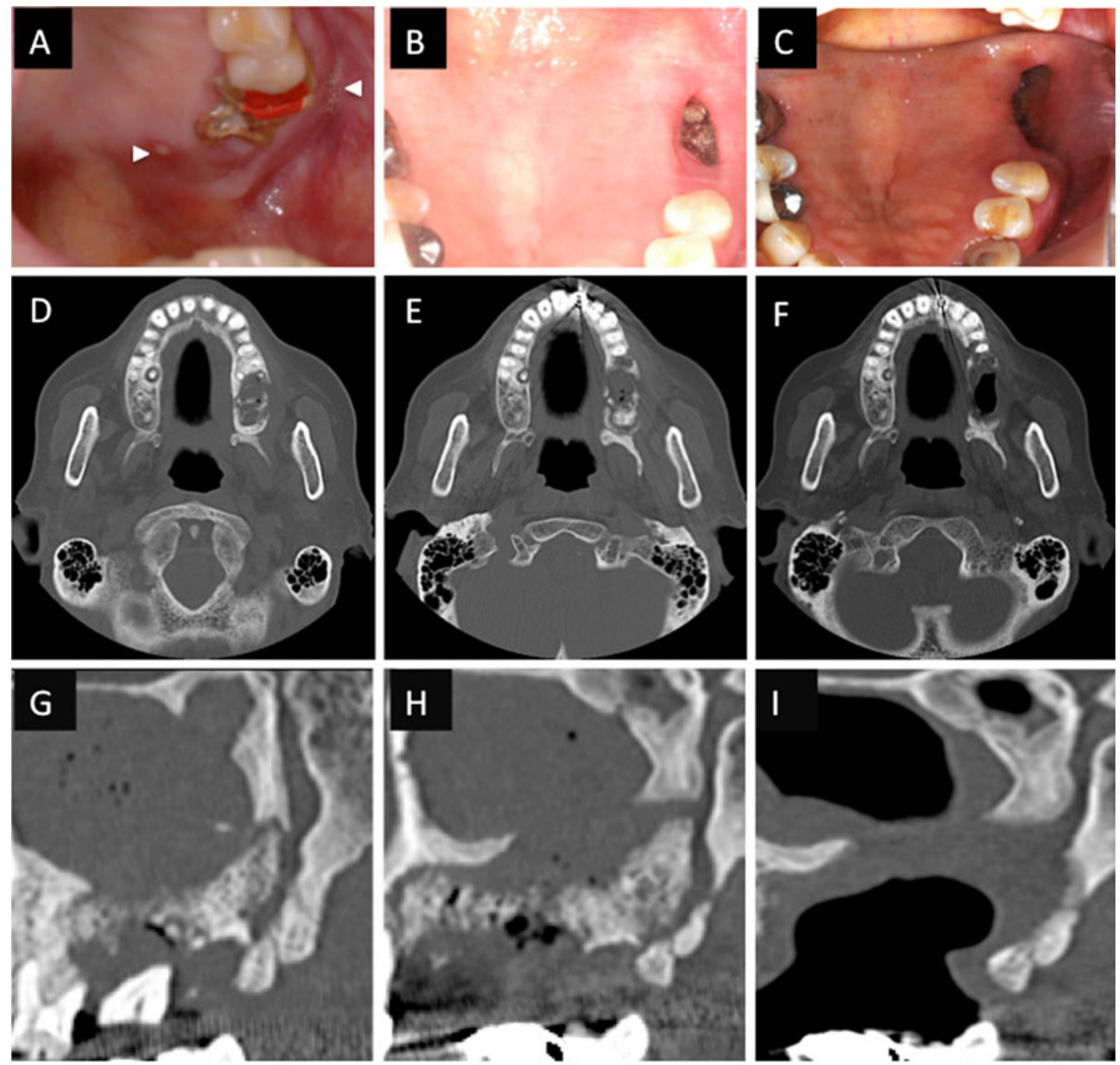

\begin{tabular}{|c|c|c|c|}
\hline $\mathrm{J}$ & At the first visit & $\begin{array}{c}\text { After 1 month of } \\
\text { teriparatide treatment }\end{array}$ & $\begin{array}{c}\text { After } 3 \text { months of } \\
\text { teriparatide treatment }\end{array}$ \\
\hline $\mathrm{P} 1 \mathrm{NP}(\mu \mathrm{g} / \mathrm{L})$ & 52.9 & 50.7 & 30.1 \\
\hline $\mathrm{s}-\mathrm{NTX}(\mathrm{nmolBCE} / \mathrm{L})$ & 27.2 & 29.8 & 38.3 \\
\hline
\end{tabular}

Fig. 1 Case 1. An 87-year-old Japanese woman with a 4-year history of alendronate therapy. a At presentation, there were multiple fistulas with purulent discharge over the left maxillary ridge (arrowheads). After 3 months of conservative therapy, the unhealed wound was surgically debrided, and two teeth were extracted. b After 12 months of conservative treatment, there was still exposed bone in the upper jaw. c After 10 weeks of teriparatide treatment, the necrotic bone had healed, and there was complete soft tissue coverage of the intraoral wound. d, g Computed tomography (CT) images showing the maxilla before tooth extraction and debridement. e, h CT images after 1 year of conservative treatment, showing expansion of the BRONJ area. f, i CT images after 10 weeks teriparatide treatment, showing improvement of the maxillary sinusitis. $\mathbf{j}$ Levels of serum N-telopeptide of type I collagen (s-NTX) and serum Nterminal propeptide of type I collagen (P1NP) reported, but the number of reports is limited and the evidence to date is mostly anecdotal [10-12]. TPTD injection is a unique pharmacological treatment for patients with primary osteoporosis. TPTD treatment stimulates bone formation and increase bone mineral density [13]. TPTD may counteract the mechanisms causing BRONJ by stimulating bone formation. An increase in the number of remodeling units and increased bone formation within each unit may promote healing and the removal of damaged bone. In case 2, the mandibular fracture and bone necrosis were successfully treated with daily TPTD injections, without the need for surgery, which is similar to the patient reported by Cheung and Seeman [8], who received the administration of 

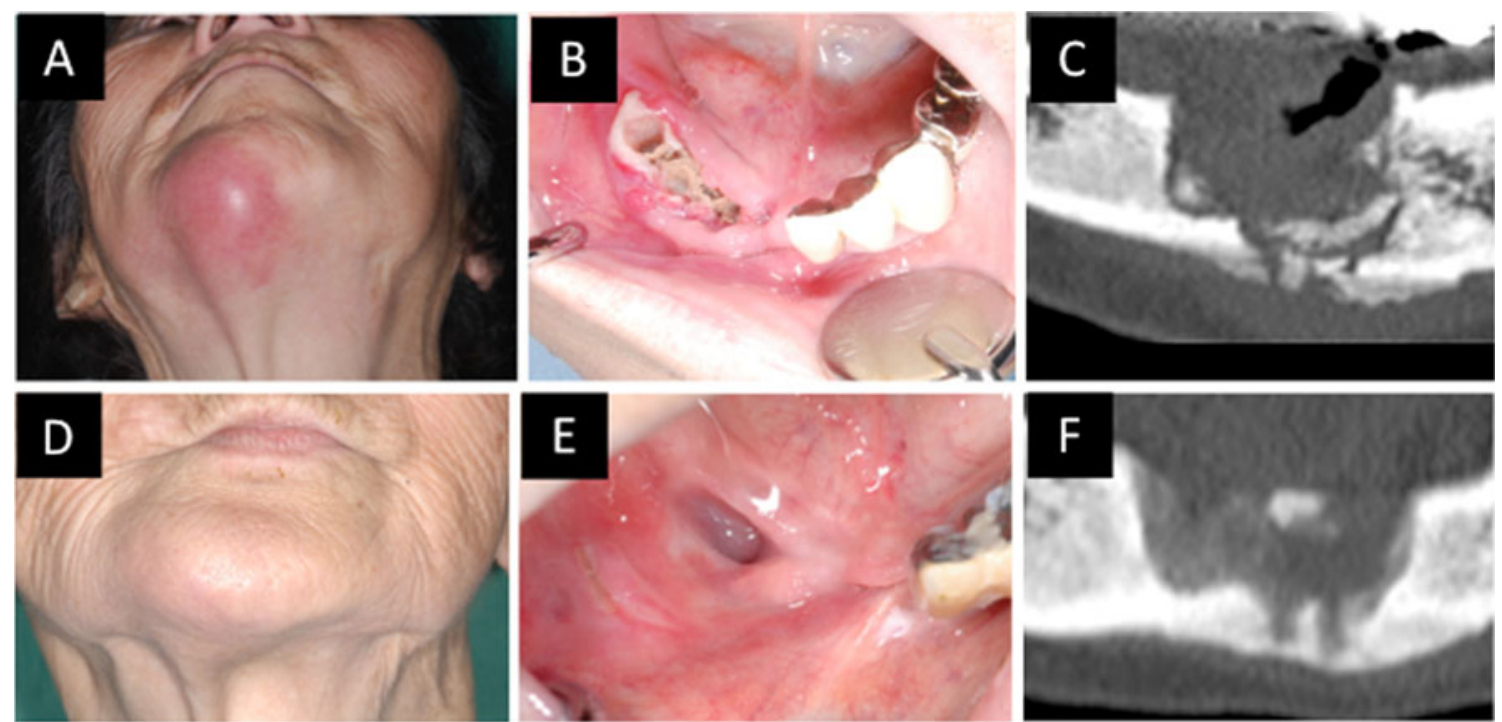

\begin{tabular}{|c|c|c|c|}
\hline $\mathbf{G}$ & At the first visit & $\begin{array}{c}\text { After 2 months of } \\
\text { teriparatide treatment }\end{array}$ & $\begin{array}{c}\text { After 4 months of } \\
\text { teriparatide treatment }\end{array}$ \\
\hline $\mathrm{P} 1 \mathrm{NP}(\mu \mathrm{g} / \mathrm{L})$ & 19.6 & 70.1 & 33.4 \\
\hline $\mathrm{s}-\mathrm{NTX}(\mathrm{nmolBCE} / \mathrm{L})$ & 15.5 & 22.9 & 32.2 \\
\hline
\end{tabular}

Fig. 2 Case 2. An 87-year-old Japanese woman with a 4-year history of alendronate therapy. a External view showing submental redness. b Intraoral view showing exposed bone after the teeth were lost. $\mathbf{c}$ CT image at presentation. d External view after 3 months of teriparatide treatment. e Intraoral view after 2 months of teriparatide treatment, showing that the necrotic bone has healed and the defect is covered with normal mucosa. f CT image after 3 months of teriparatide treatment. g Levels of serum N-telopeptide of type I collagen (s-NTX) and serum N-terminal propeptide of type I collagen (P1NP)

TPTD for osteonecrosis of the jaw in association with alendronate therapy. In both our patients, TPTD treatment was effective and achieved soft tissue coverage of exposed bone. This is the first report describing successful treatment of BRONJ with weekly TPTD injections.

In conclusion, the outcomes of the cases presented suggest that weekly TPTD injections can be effective for the treatment of stage 3 BRONJ. If weekly and daily TPTD injections are both effective, we can choose the TPTD treatment regimen according to the condition of the patient. In our patients, stage 3 BRONJ was successfully treated by either weekly or daily TPTD injections, thereby improving their quality of life. This indicates that weekly TPTD injections might result in resolution of stage 3 BRONJ by increasing the rate of bone remodeling. Our data indicate that when it is determined that a stage 3 BRONJ patient's condition does not improve under conservative therapy and there are no other medical contraindications, daily, or weekly TPTD treatment should be considered. Our data also suggest that it may now be appropriate to initiate limited investigation of the response to weekly PTH treatment, of uncomplicated stage 2 BRONJ cases with persistent bare bone.

\section{Conflicts of interest None.}

Open Access This article is distributed under the terms of the Creative Commons Attribution Noncommercial License which permits any noncommercial use, distribution, and reproduction in any medium, provided the original author(s) and the source are credited.

\section{References}

1. Franken AA, van Blijderveen NJ, Witjes MJ, Netelenbos CJ (2011) Bisphosphonate-related osteonecrosis of the jaw. Ned Tijdschr Geneeskd 155:A3077

2. Ruggiero SL, Dodson TB, Assael LA, Landesberg R, Marx RE, Mehrotra B (2009) American Association of Oral and Maxillofacial Surgeons position paper on bisphosphonate-related osteonecrosis of the jaws - 2009 update. J Oral Maxillofac Surg 67:2-12

3. Nakamura T, Sugimoto T, Nakano T, Kishimoto H, Ito M, Fukunaga M, Hagino $\mathrm{H}$, Sone T, Yoshikawa H, Nishizawa Y, Fujita T, Shiraki M (2012) Randomized Teriparatide [Human Parathyroid Hormone (PTH) 1-34] Once-Weekly Efficacy Research (TOWER) trial for examining the reduction in new vertebral fractures in subjects with primary osteoporosis and high fracture risk. J Clin Endocrinol Metab 97(9):3097-3106

4. Reid IR, Cornish J (2011) Epidemiology and pathogenesis of osteonecrosis of the jaw. Nat Rev Rheumatol 8:90-96 
5. Kyrgidis A, Vahtsevanos K (2010) Osteonecrosis of the jaw in patients receiving oral bisphosphonates. Osteoporos Int 21:535536

6. Woo SB, Hellstein JW, Kalmar JR (2006) Narrative [corrected] review: bisphosphonates and osteonecrosis of the jaws. Ann Intern Med 144:753-761

7. Narongroeknawin P, Danila MI, Humphreys LG Jr, Barasch A, Curtis JR (2010) Bisphosphonate-associated osteonecrosis of the jaw, with healing after teriparatide: a review of the literature and a case report. Spec Care Dent 30:77-82

8. Cheung A, Seeman E (2010) Teriparatide therapy for alendronateassociated osteonecrosis of the jaw. N Engl J Med 363:2473-2474

9. Lau AN, Adachi JD (2009) Resolution of osteonecrosis of the jaw after teriparatide [recombinant human PTH-(1-34)] therapy. J Rheumatol 36:1835-1837
10. Lee JJ, Cheng SJ, Jeng JH, Chiang CP, Lau HP, Kok SH (2011) Successful treatment of advanced bisphosphonate-related osteonecrosis of the mandible with adjunctive teriparatide therapy. Head Neck 33:1366-1371

11. Kyrgidis A, Antoniades K (2011) Could teriparatide be the treatment for osteonecrosis of the jaws? Head Neck 33:1382-1383, Author reply, 1383

12. Harper RP, Fung E (2007) Resolution of bisphosphonateassociated osteonecrosis of the mandible: possible application for intermittent low-dose parathyroid hormone [rhPTH(1-34)]. J Oral Maxillofac Surg 65:573-580

13. Jiang Y, Zhao JJ, Mitlak BH, Wang O, Genant HK, Eriksen EF (2003) Recombinant human parathyroid hormone (1-34) [teriparatide] improves both cortical and cancellous bone structure. J Bone Miner Res 18:1932-1941 